\title{
Essais de stabilité effectués sur un groupe hydroélectrique
}

\author{
Stability tests on a hydroelectric unit
}

\author{
PAR MM. LAURENT ET FAVEZ
}

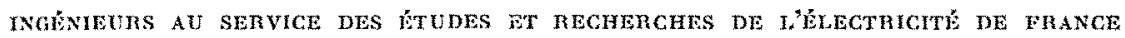

\begin{abstract}
Le but des essais est de vérifier les lois de régulation des groupes hydro-électriques en mesurant la stabilité. — Rappel des modalitís de fonctionnement d'un régulateur. - Examen de sept caractéristiques du réglage, calcul de quatre d'entre elles et mesure des trois autres. Application de l'analyse harmonique $\dot{a}$ l'étude du comportement du groupe, au moyen de pulsations sinusoïdales propoqués ef engendrant des oscillations dans divers éléments de la chaîne de réglage: a) dans l'ensemblt" intérieur an régulatenr; b) dans la seconte partie de la chaîne, extérieure au régulatenr. - Etude de l'influence, sur la stabilité, des retards observés. Conclusion: les équations de la régulation sont vérifiées, sauf en ce quii concerne le servo-moteur, qui devra faire l'objet d"essais ultérieurs.
\end{abstract}

The purpose of the tests is to check hydroelectric turbine governor laws by measuring their stability. Recapitulation of the methods used in governors. An examination of seven governor characteristics, four of them calcula: ed, three measured. The application of harmonic analysis to the study of the behavionr of a nnit by setting up sinusoidal pulses which canse oscillations in barious parts of the governor system

a) within the governor itself

b) in the second part of the system, ontside the governor proper. An investigation of the effect of the delaye observed on stability.

Conclusion: The governor equations are verified except where they affect the servomotor which will be subjected to further tests.

\section{I. - GENERALITES}

\section{Notations}

$\Delta \omega=\frac{\Omega-\Omega_{0}}{\Omega_{0}}$ : écart relatif de vitesse du groupe;

$\Delta \omega_{0}$ : écart perturbateur (en valeur relative) déterminé par étalonnage du variateur de vitesse. Cet écart est complé positivement s'il tend à augmenter la fréquence en régime statique;

$\Delta a$ : somme des écarts (relatifs) tachymétrique et accélérométrique;

$\Delta z$ : ćlongation du pointeau et du piston du servo-moteur de distribution d'huile au vannage (valeur ramenée à un écart de vitesse);

$\Delta x=\frac{x-x_{0}}{x_{0}}$ : élongation relative du vannage;

$\Delta p_{0}$ : écart relatif de puissance (en dehors du coup de bélier);

$\Delta p$ : écart relatif de puissance (en tenant compte du coup de bélier);

$\Delta h$ : écart relatif de pression dâ au coup de bélier;

$\Delta u$ : écart relatif de tension;

$m$ : dosage accélérométrique; $\alpha$ : coefficient global d'autoréglage;

$\tau_{y}^{\prime}$ : lenteur en course du vannage;

$\mathrm{K}$ : inverse de la pente de la courbe puissance-ouverture en pales bloquées;

$j:$ coefficient de rendement;

$\lambda:$ pulsation de l'oscillation perturbatrice;

$\Theta$ : temps caractéristique du coup de bélier;

$\mathrm{T}$ : inertie des masses tournantes.

\section{CARACTERISTIQUES DU GROUPE ETUDIE}

TUnbine (type Kaplan):

Hauteur de chute au cours des essais...... $17 \mathrm{~m}$ Puissance sous une chute nette de $17 \mathrm{~m} \ldots 55300 \mathrm{ch}$ Vitesse ............................ $\quad 93,75 \mathrm{tr} / \mathrm{mn}$

Régulateur : type accélérotachymétrique.

Alternateur :

Puissance .................... $43000 \mathrm{kVA}$

Tension nominale ............... $10 \mathrm{kV}$

Régulateur de tension : type continu...., $12.10^{\alpha} \mathrm{kg}-\mathrm{m}^{2}$ détecteur par pont à lampes; amplification par amplidyne et survolteur. 


\section{A) But des essais :}

Les essais ont été réalisés principalement en vue de vérifier les lois qui régissent la régulation des groupes hydroélectriques. Leur mise en équation a fait l'objet d'un certain nombre de notes théoriques qui, à notre connaissance, n'ont pas reçu jusqu'ici de confirmation expérimentale tant au point de vue d'un régulateur propre que du fonctionnement de plusieurs régulateurs en parallèle.

L'importance pratique de ces équations n'est cependant pas négligeable : si celles-ci sont vérifiées, il suffira en effet de mesurer quelques paramètres pour pouvoir obtenir la stabilité d'un groupe et, partant, la stabilité d'un réseau.

Notre but était donc de mesurer la stabilité et de vérifier les équations :

$1^{\circ}$ d'un groupe fonctionnant seul;

$2^{\circ}$ de plusieurs groupes fonctionnant en parallèle.

Malheureusement, le second point n'a pu être étudié en raison des exigences de l'exploitation; nous avons pu cependant examiner le fonctionnement complet d'un groupe dans' différents cas.

Le groupe étudié était un groupe Kaplan de la Centrale d'Ottmarsheim, d'une puissance nominale de l'ordre de $40 \mathrm{MW}$. Les essais, qui ont été effectués à $15 \mathrm{MW}, 25$ et $35 \mathrm{MW}$, ont donc à peu près couvert toute la plage de puissance de la machine.

Ce groupe était muni d'un régulateur du type accélérotachymétrique. Il avait un régulateur de tension à amplidyne et survolteur qui, chose particulière en France, comportait un asservissement de tension à la fréquence. Cet asservissement pouvait varier d'une façon continue entre les valeurs 0 (asservissement nul) et 1 (à $1 \%$ de variation de fréquence correspond $1 \%$ de variation de tension).

\section{B) Rappel sur le fonctionnement du régu- lateur :}

Le régulateur se compose d'un accéléromètretachymètre qui reçoit le courant d'un alternateur pilote (fig. 1). La somme des écarts accélérométrique et tachymétrique agit sur un tiroir de distribution d'huile qui fait varier la position du vannage, donc la puissance du groupe. Si celuici est coupé du réseau et fonctionne, par exemple, sur une résistance liquide ou à vide, cet écart de puissance va produire une modification de la vitesse de la machine qui dépend de l'inertie de l'alternateur et du coefficient d'autoréglage (ce coefficient mesure la variation de la charge motrice avec la vitesse).

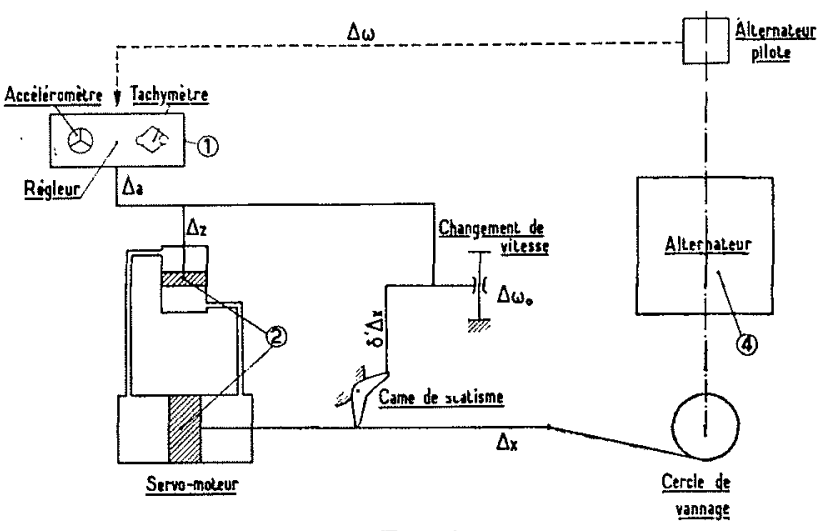

Fig. 1

Schéma d'un régulateur accélérotachymétrique.

Le schéma fonctionnel de ce dispositif est reproduit sur la figure $n^{\circ} 2$. Supposons un écart de vitesse $\Delta \omega\left(^{\star}\right)$. La somme des effets tachymétrique et accélérométrique, soit $\Delta a$, peut être conjuguée avec un écart imprimé par le changement de vitesse, soit $\Delta \omega_{0}\left({ }^{\star \star}\right)$.

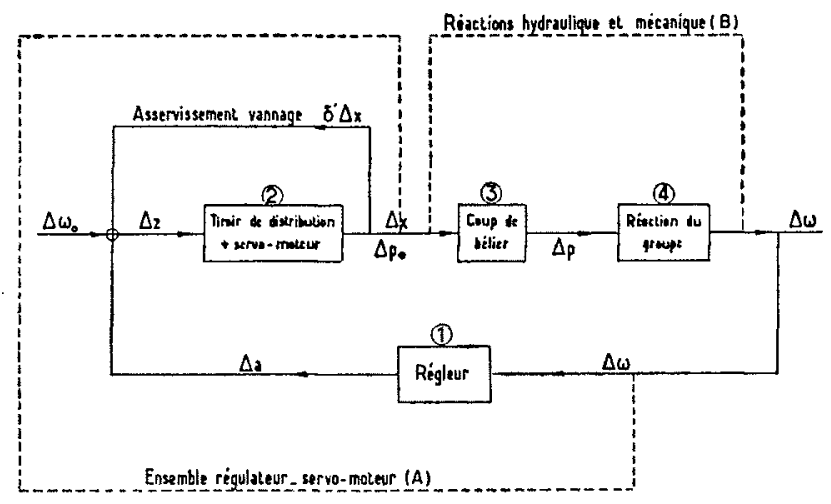

Fig. 2

Schéma fonctionnel d'un régulateur accélérotachymétrique.

Ces écarts agissent sur l'ensemble du servomoteur qui les transforme en une variation $\Delta x$ du vannage, à laquelle correspond une variation $\Delta p_{0}$ de puissance déterminée par la pente $1 / \mathrm{K}$ de la courbe «puissance ouverture ». Par suite de l'existence du coup de bélier, à cet écart $\Delta p_{0}$ va correspondre un écart réel de puissance $\Delta p$, légèrement déphasé par rapport à $\Delta p_{0}$.

A cet écart $\Delta p$, le groupe va réagir par son inertie et son coefficient d'autoréglage en faisant varier sa vitesse de $\Delta \omega$.

La chaîne de régulation se compose donc de quatre éléments. Nous allons rappeler rapidement les lois qui les régissent :

(*) Les notations sont indiquées au début de la note.

$\left.{ }^{(*}\right) \mathrm{Au}$ cours de cet exposé, il n'a pas été tenu compte du statisme, les essais ayant été réalisés sur résistance liquide sans asservissement fréquence-puissance. 
- Le premier élément représente la somme des écarts tachymétrique et accélérométrique :

$$
\Delta a=\Delta \omega+m \frac{d}{d t} \Delta \omega
$$

\section{- Equation du deuxième élément :}

La figure 1 montre qu'à un écart $\Delta z$ du servomoteur correspond un flux d'huile, par conséquent une vitesse du vannage, ce qui se traduit par la relation :

$$
\frac{d}{d t} \Delta x=-\frac{1}{\tau_{x}^{\prime}} \Delta z
$$

où $\tau_{;}^{\prime}$ caractérise la lenteur de réponse du vannage.

Le signe - indique qu'à un écart de vilesse positif doit correspondre une fermeture du vannage.

- Le troisième élément représente l'influence du coup de bélier, lorsque le vannage est soumis à une oscillation. On montre que l'écart relatif de pression est défini par la relation :

$$
\Delta h=-\Theta \frac{d}{d t}\left(\frac{\Delta p_{0}}{j}+\frac{1}{2} \Delta h\right)
$$

- La quatrième équation est la traduction de l'équation des masses' tournantes:

$$
\mathrm{I} \frac{d}{d t} \Delta \omega=\mathrm{C}_{m}-\mathrm{C}_{r}
$$

On a l'habitude d'écrire cette relation sous la forme :

$$
\frac{d}{d t} \Delta \omega=\frac{1}{\mathrm{~T}}(\Delta p-\alpha \Delta \omega)
$$

où $\mathrm{T}$ caractérise l'inertie des masses tournantes.

On peut, à partir de ces quatre équations, étudier les oscillations de vitesse, en éliminant les autres variables; ceci conduit à une équation du troisième ordre à coefficients constants. Mais, si l'on accepte une légère variation des coefficients, on peut admettre la forme suivante :

$$
\frac{d^{2} \Delta \omega}{d t}+\mathrm{P} \frac{d}{d t} \Delta \omega+\mathrm{Q} \Delta \omega=0
$$

où les coefficients $\mathrm{P}$ et $\mathrm{Q}$ dépendent de la pé- riode des oscillations, mais varient peu avec celle-ci.

Si ces équations sont vérifiẻes, il suffira de connaître les coefficients de réglage : $m, \tau_{m}{ }^{\prime}, \mathrm{K}, j$, $\alpha$ et $T$ pour obtenir l'équation. Les mesures de ces caractéristiques étant assez simples, il serait dans ce cas relativement aisé d'évaluer la stabilité d'un groupe fonctionnant seul.

Pour vérifier ces équations, on a utilisé la méthode de l'analyse harmonique. Elle consiste à faire osciller sinusoïdalement la vitesse du groupe et à étudier en fonction de chaque pulsation la réponse des différents éléments. Les équations précédentes peuvent s'écrire, en régime harmonique :

$$
\begin{aligned}
\frac{\Delta a}{\Delta \omega} & =1+m i \lambda \\
\frac{\Delta x}{\Delta z} & =-\frac{1}{\tau^{\prime} i \lambda} \\
\frac{\Delta p}{\Delta p_{0}} & =\frac{1-(i \lambda \Theta / 2)[(3 / j)-1]}{1+i(\lambda \Theta / 2)} \\
\frac{\Delta \omega}{\Delta p} & =\frac{1}{i \lambda \mathrm{T}+\alpha}
\end{aligned}
$$

Dans les études de régulation, il est commode de traduire ces relations par des courbes tracées en coordonnées «gain-phase »: on porte en abscisse le logarithme de la pulsation et en ordonnée le logarithme du gain multiplié par 20, ce qui donne des décibels, et la phase en degrés.

Par conséquent, pour vérifier ces équations, nous allons, d'une part, tracer la courbe théorique calculée à partir des coefficients $m, \tau_{x}^{\prime}$, etc., et, d'autre part, par la méthode de l'analyse harmonique, étudier le gain et le déphasage introduits par chaque élément. La comparaison des diagrammes permettra de contrôler la validité des équations.

Les essais peuvent donc se diviser en deux parties :

Première partie : étude des caractéristiques $d u$ réglage : $m$, $\tau_{a}^{\prime}$, etc., pour établir les courbes théoriques.

- Deuxième partie : étude du comportement du groupe par l'analyse harmonique.

\section{II. - ETUDE DES CARACTERISTIQUES DU REGLAGE}

Les caractéristiques de rẻglage sont :

- I.e dosage accélérométrique $m$;

- La lenteur de réglage $\tau_{x}^{\prime}$;
- La sensibilité de l'installation au coup de bélier, $\Theta$;

- L'inertie des masses tournantes, $T$; 
- Le coefficient d'autoréglage $\alpha$;

- La pente $1 / \mathrm{K}$ de la courbe «puissance-ouverture $\gg$;

- Le coefficient de rendement, $j$.

\section{A) Calcul des caractéristiques $\mathrm{T}, \Theta, \mathrm{K}$ et $j$ :}

Certaines de ces caractéristiques peuvent se déterminer par le calcul :

$1^{\circ}$ Le coefficient $T$, qui représente l'inertie des masses tournantes, peut se calculer à partir du $\mathrm{PD}^{2}$ et de la vitesse de rotation de l'alternateur;

$2^{\circ}$ Le coefficient $\Theta$ qui caractérise l'inertie des masses hydrauliques, peut se déduire du volume et de la vitesse de l'eau contenue dans les tuyauteries d'alimentation de la turbine;

$3^{\circ}$ Les coefficients $\mathrm{K}$ et $j$ se déterminent à partir de la courbe puissance-ouverture et de la courbe de rendement.

B) Mesure des caractéristiques $\tau_{x}^{\prime}, m$ et $\alpha$ :

1. Lenteur de Réglage. - Considérons l'équation (2); si l'on imprime au tiroir un écart $\Delta z$, il suffira de mesurer la vitesse de variation du vannage pour en déduire $\tau_{x}^{\prime}$.

Considérons maintenant le schéma (2) et sup-
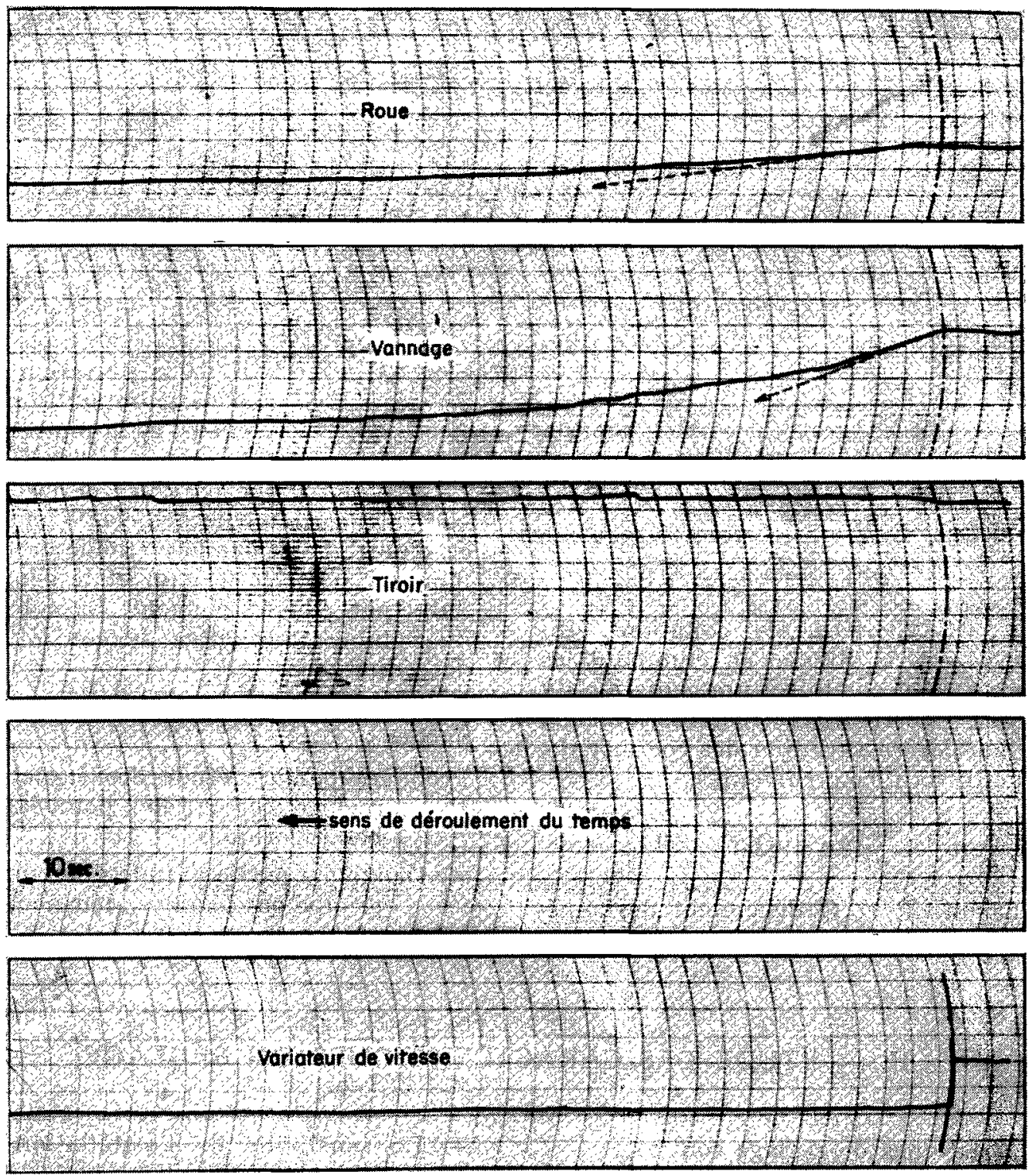

Fic. 3. - Mesure des promptitudes de la roue et du vannage (Groupe sur réseau. Statisme $4 \%$. Puissance initiale $15 \mathrm{MW}$ ). 
posons la vitesse constante, soit $\Delta \omega=0$. Pour cela, il suffit de garder le groupe couplé sur le réseau; on obtient dans ce cas $\Delta \omega_{0}=\Delta z$. Il suffit donc d'imprimer, à l'aide du changement de vitesse, un écart $\Delta \omega_{0}$ et de mesurer la vitesse du vannage correspondante.

La figure (3) donne un exemple de la méthode de mesure. L'écart $\Delta \omega_{0}$ esl lu directement; la vitesse du vannage se déduit de la mesure de la pente de la courbe à l'origine (cette pente varie ensuite à cause du statisme).

Les résultats des différentes mesures sont rassemblés dans la figure 4.

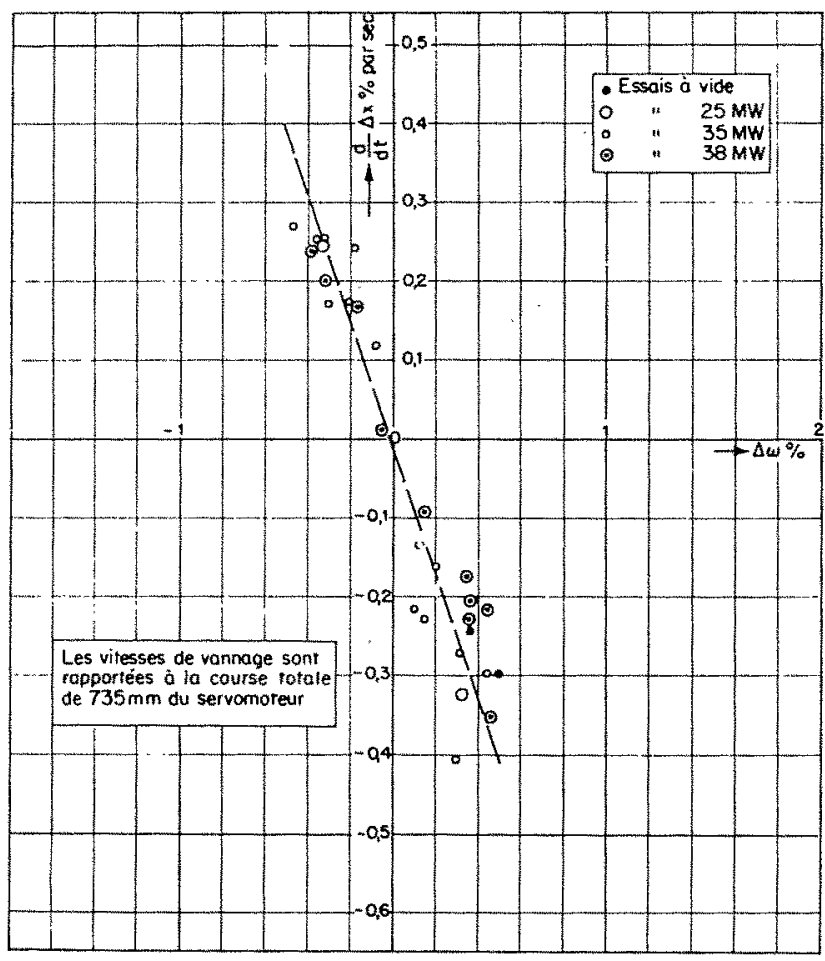

Fig. 4

Mesure de la promptitude du vannage.

Nous avons porté en abseisses l'écart $\Delta \omega_{0}$. Le coefficient $\tau_{z}^{\prime}$ est mesuré par la pente de la courbe.

2. DOSAGE ACCÉLÉROMÉTRIQUE $m$. - Le groupe ne peut plus débiter sur le réseau, en raison de la nécessité de créer des variations de fréquence. On va donc fonctionner soit sur une résistance liquide, soit, plus simplement, à vide. On donne un à-coup, toujours à l'aide du changement de vitesse. L'écart du tiroir de distribution $\Delta z$ peul s'écrire :

$$
\Delta z=\Delta \omega+m \frac{d}{d t} \Delta \omega-\Delta \omega_{0}
$$

Si l'on considère cette équation au moment où le tiroir de distribution a une élongation nulle, on peut écrire :

$$
m=-\frac{\lambda \omega-\lambda \omega}{(d / d t)\lrcorner \omega}
$$

Par conséquent, en relevant la courbe des écarts de vitesse, on peut, à l'aide de la mesurc d'une tangente, déterminer le coefficient accéléronétrique (fig. 5 ).

Les mesures, bien que peu précises, ont domnć un coefficient accélérométrique égal à 2,5 secondes, contre 2,7 secondes, valeur déterminée avec plus de précision dans les ateliers du constructeur.

3. Coffficient d autoréglage $\alpha$, Ce coefficient est égal à la différence entre les coefficients $\alpha_{n n}$ et $\alpha_{r}$, qui caractérisent les réactions du couple moteur et du couple résistant en fonction de la fréquence (fig. 6).

La courbe de couple moteur a été fournie par le constructeur à partir des courbes des essais d'emballement sur modèle réduit (ces essais ont été faits en fonction de la vitesse). La pente de la courbe donne le coefficient $\alpha_{m}$.

Pour déterminer $\alpha_{p}$, nous remarquerons que les essais ont été réalisés sur résistance liquide. La puissance débitée par le groupe était de la forme :

$$
P=\frac{U^{2}}{R}
$$

On en déduit que $\Delta p / \Delta \omega$ pour les petits écarts est égal à $2(\Delta u / j \omega)$, et $\Delta c / \Delta \omega$, c'est-à-dire la variation du couple résistant est égale à :

$$
2(\Delta u / \Delta \omega)-1
$$

Par conséquent, il suffit de connaitre la variation de la lension en fonction de l'écart de fréquence.

On a relevé dans ce but les variations de lit tension au cours des différents essais en sollicitations sinusoïdales. Les figures 7 et 8 montrent les résultats obtenus avec régulateur de tension asservi et en excitation bloquée.

Le coefficient $\alpha_{r}$ est à peu près constant aux différentes périodes de la sollicitation. Les divergences entre les courbes de la figure 8 sont dues à ce que la tension n'était pas la même au cours des essais. En effet, on a avantage à opérer à la tension la plus basse possible pour éviler des amorcages de la résistance liquide. 

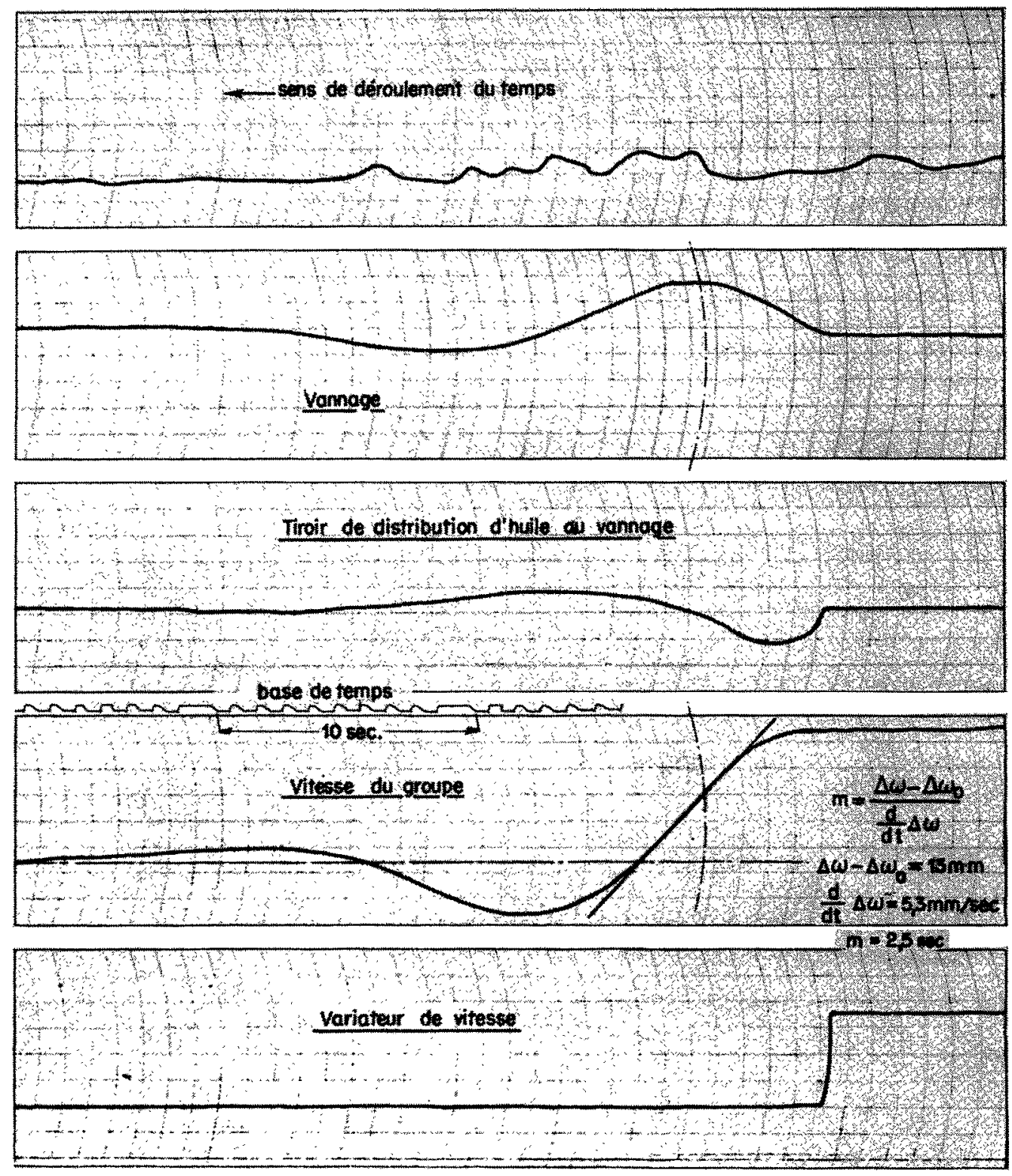

FIG, 5 ,

Mesure du dosage accélérométrígue.

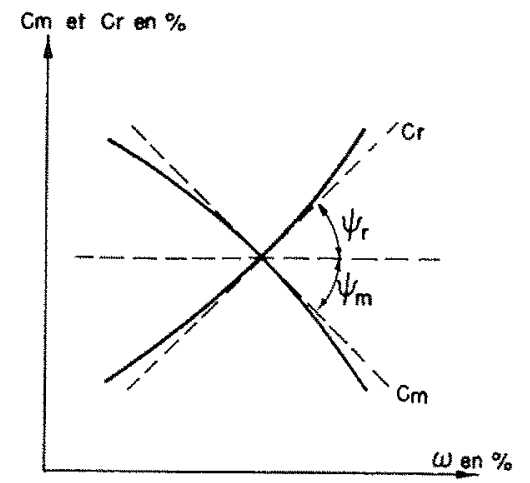

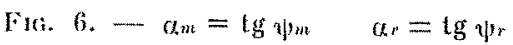

Courbes représentant les variations des couples résistant et moteur en fonclion de la fréçuence. 


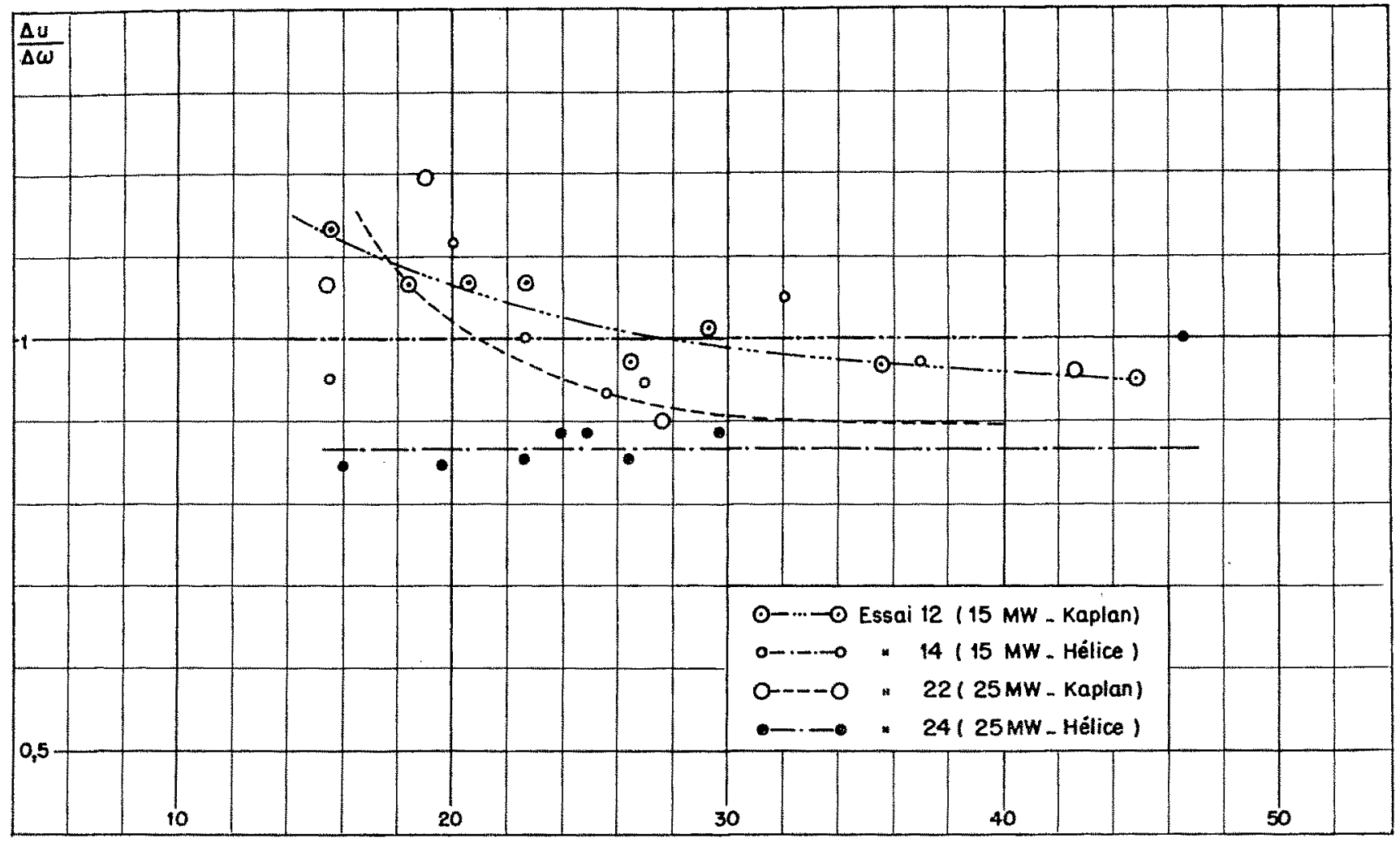

Période de l'oscillation pertubatrice en secondes

Frg. $7 .-$ Valeurs du rapport $\Delta u / \Delta \omega$. Asservissement maximum tension-fréquence.

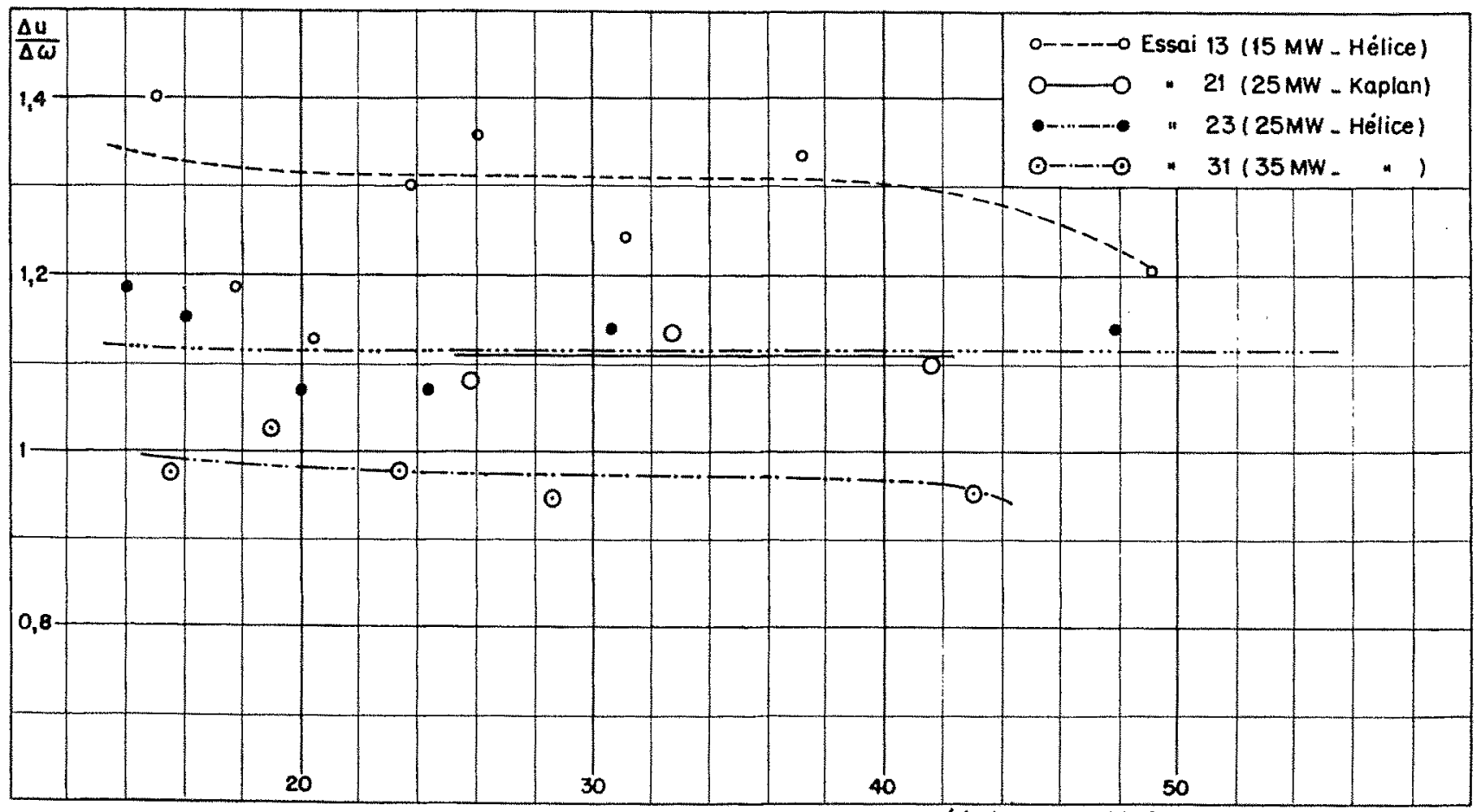

Période de l'oscillation pertubatrice en secondes

Fic. 8. - Valeurs du rapport $\Delta_{\text {ata }}$. Régulateur de lension bloqué. 


\section{III. - ETUDE DU COMPORTEMENT DU GROUPE PAR L'ANALYSE HARMONIQUE}

Pour obtenir des pulsations sinusödales du groupe, on a agi sur le variateur de vitesse, et tous les éléments de la chaine de réglage ont subi des variations sinusoïdales, comme le montre la figure 9 oi l'on retrouve de bas en haut:

- L'oscillation inductrice $\Delta \omega_{0}$;

-.. L'oscillation de vitesse $\Delta \omega$;

L'oscillation du tiroir de distribution d'huile au vannage $\Delta z$;

...- L'oseillation de vannage $\Delta x$;

L'oscillation de tension $\Delta u$.
Ia premicre est évidemment sinusoïdale par définition, puisqu'engendrée directement par le variateur de vitesse. Mais on voit que les différents éléments de la chaîne répondent eux aussi de façon sinusoïdale.

A partir de ces resultats, on peut mesurer : - Soit le gain de chaque élément, c'est-à-dire les rapports $\Delta x / \Delta z$ et $\Delta \omega / \Delta x$ puisqu'on a mesuré la frécquence et le vannage. Cette méthode permet de déterminer la fonction relative à chaque élément.

- Soit le gain global de la boncle $\Delta \omega / \Delta \omega_{4}$.
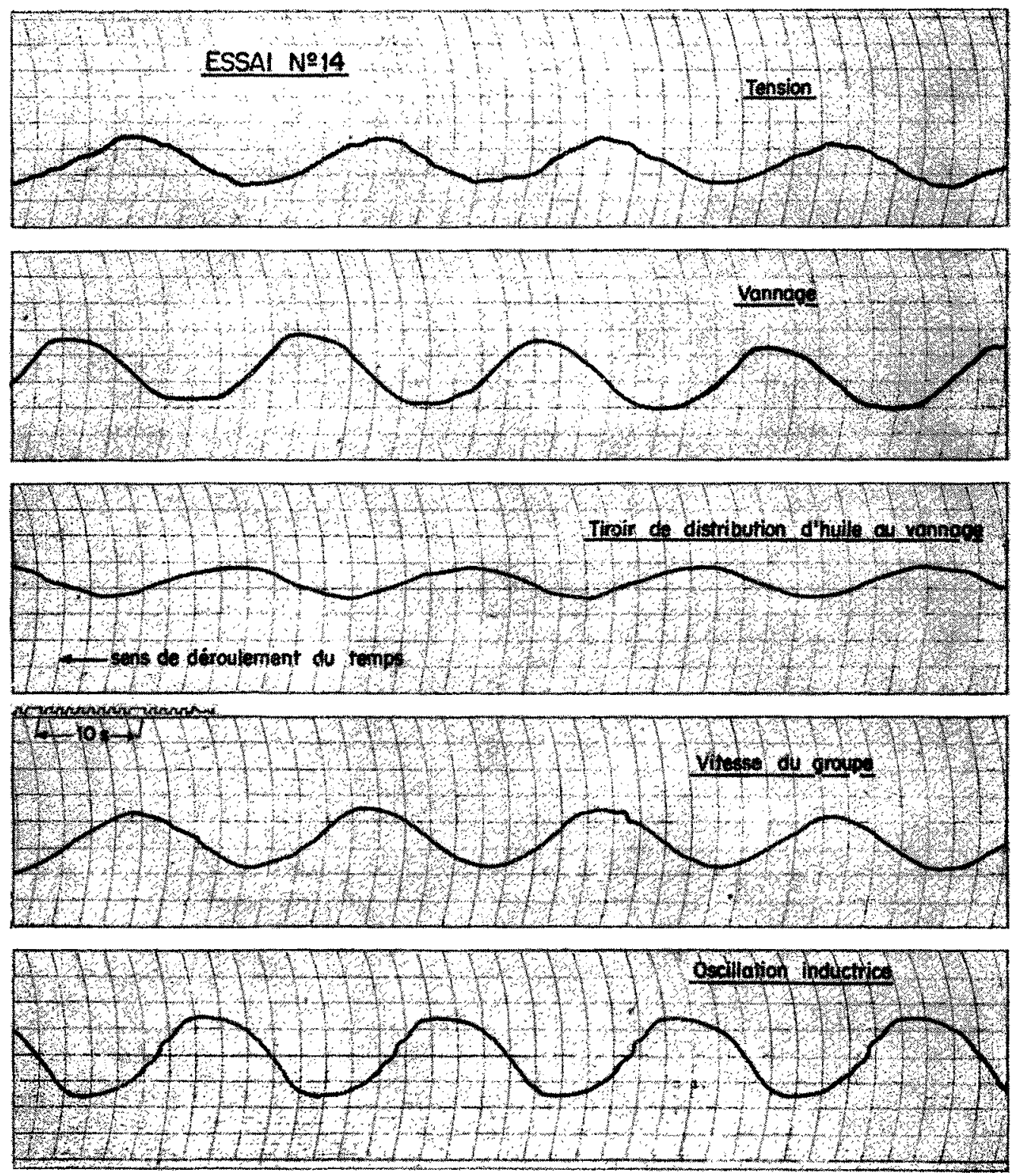

Fir. 9 
La première méthode conduit à étudier chaque élément séparément et à contròler s'il répond bien aux équations, tandis que la méthode glo-

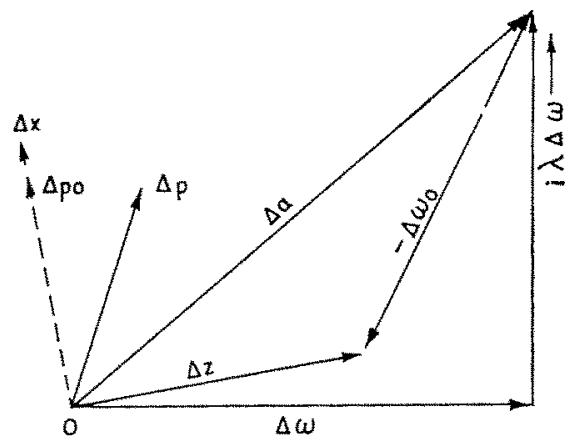

Fik, 10. -... Construction du vecteur $\Delta z$. bale revient à déterminer directement la stabilité du groupe.

Le but des essais étant principalement de vérifier les différentes équations de la régulation, nous avons étudié chaque élément séparément.

Les mesures effectuées ont permis de décomposer la chaine en différentes parties (fig. 2) :

1" L'ensemble intérieur au régulateur, qui comprend le régleur et le servo-moteur jusqu'au vannage : nous l'appellerons la partie A;

$2^{\circ}$ Une partie $B$, exlérieure an régulateur, qui tient compte du coup de bélier et de la réaction des masses tournantes.

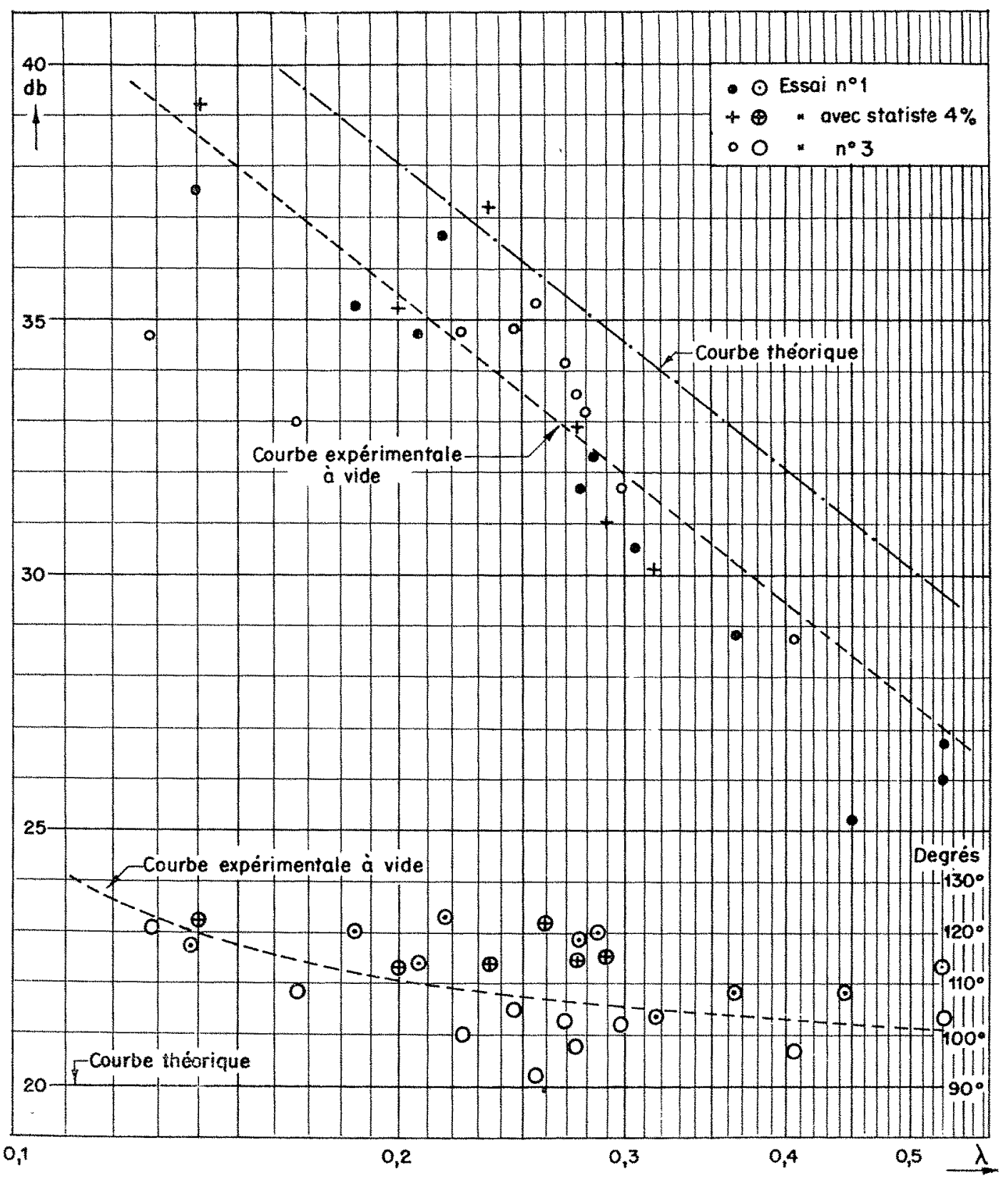

Fif. 11

Gain of dephasage du servo-moteur. Essatis a vide. 
Frg. 12

Gain et déphasage du servo-moteur. Essais à $15 \mathrm{MW}$

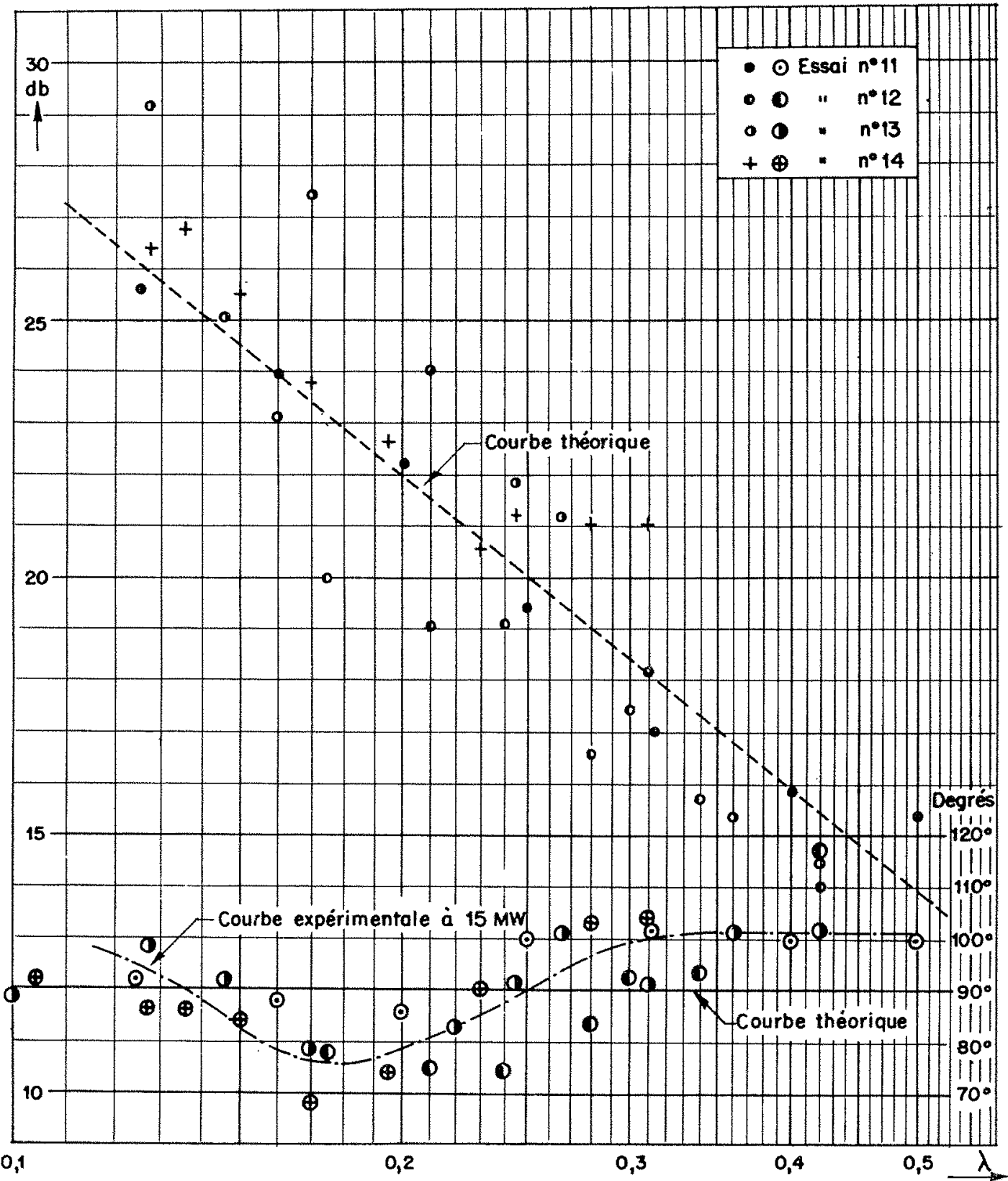

Nous allons étudier successivement ces deux parties.

\section{A) Etude de l'ensemble intérieur au régu- lateur :}

La grandeur de sortie du régleur $\Delta a$ représente une variation de flux d'huile, qu'il n'est pas possible de mesurer sur place. Le régleur est, par contre, étudié en atelier. Nous pouvons donc admettre son bon fonctionnement.

Les performances du servo-moteur sont caraclérisées par le rapport $\Delta x / \Delta z$.

En régime sinusoïdal, les différentes grandeurs peuvent être reportées sur un diagramme vectoriel construit de la façon suivante (fig. 10):

Considérons un écart de vitesse $\overrightarrow{\Delta \omega}$ suivant l'équation (1); la somme des écarts tachymétrique $\overrightarrow{\Delta \omega}$ et accélérométrique $m(d / t) \overrightarrow{\Delta \omega}$ donne $\overrightarrow{\Delta a}$ auquel on ajoute $\overrightarrow{\Delta \omega_{0}}$ pour obtenir le mouvement du tiroir $\overrightarrow{\Delta z}$.

D'autre part, l'équation (2) montre que le recteur $(d / d t) \overrightarrow{\Delta x}$, perpendiculaire à $\overrightarrow{\Delta x}$, est proportionnel à $\overrightarrow{\Delta z}$. On en déduit que l'écart de vannage doit être en avance de $90^{\circ}$ sur l'écart du tiroir.

A cet écart $\overrightarrow{\Delta x}$ correspond un écart proportion- 


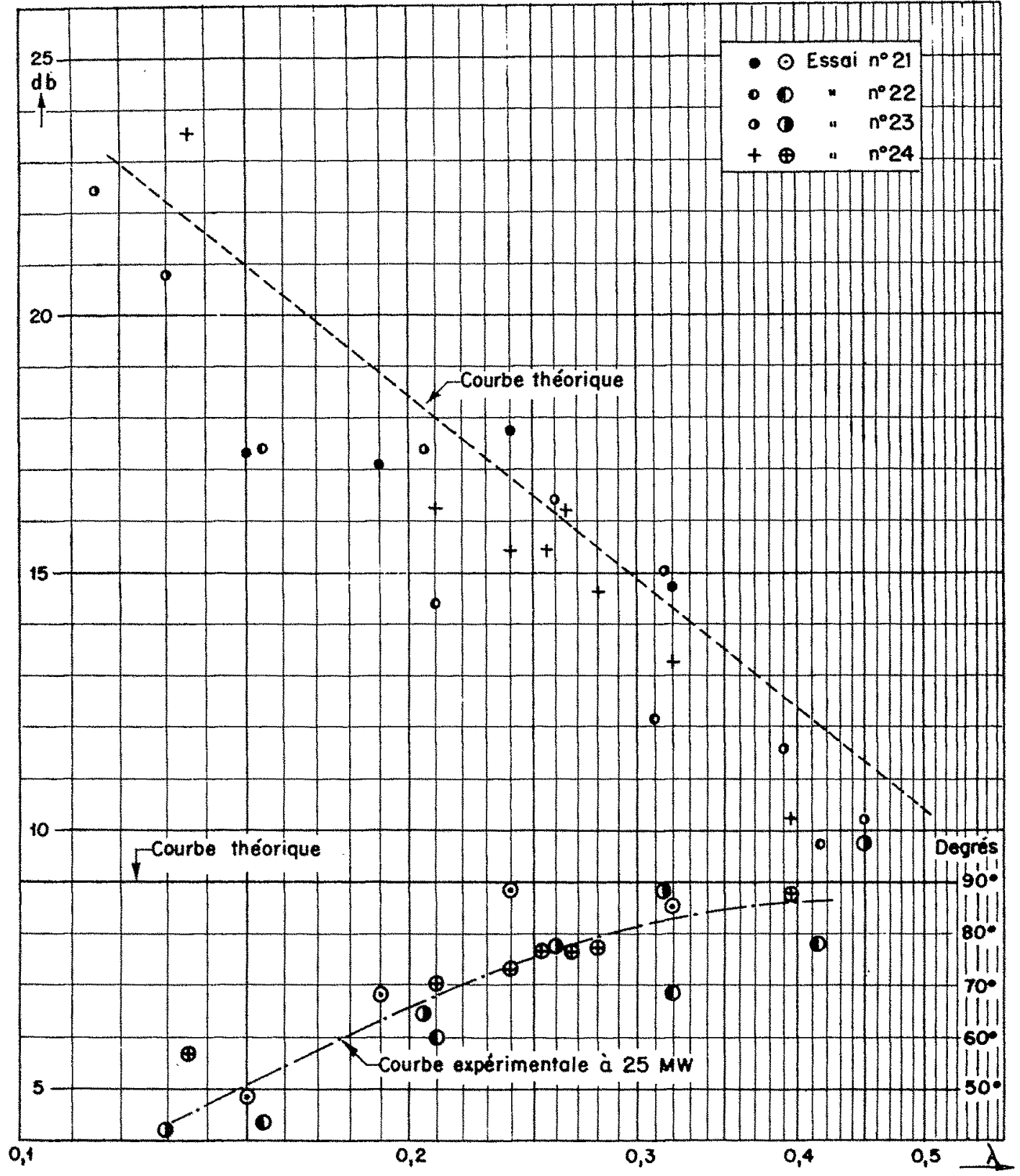

FIG. 13

Gain et déphasage du servo-moteur. Essais a $25 \mathrm{MTW}$. nel $\overrightarrow{\Delta p_{0}}$. Puis le coup de bélier transforme l'écart de prissance $\overrightarrow{\Delta p_{0}}$ en un ecart $\overrightarrow{\Delta p}$ et introduit un déphasage. Et nous retombons, par l'intermsdiaire de l'équation (4), sur l'écart $\overrightarrow{\Delta \omega}$.

Le rapport $\Delta x / \Delta z$ est donc caractérisé :

$1^{\circ}$ par un déphasage de $90^{\circ}$;

$2^{\circ}$ par un module inversement proportionnel à la pulsation, ce qui va se traduire, en coordonnées logarithmiques, par une droite descendante.

Les figures 11 à 14 montrent les résultats d'essai :
- La figure 11 représente les résultats a vide. En haut se trouve la courbe de gain : e'est une droile descendante.

La courbe théorique, déduite du coefficient $\tau^{\prime}$ déterminé plus haut, se trouve au-dessus de la courbe expérimentale.

Il y a entre les deux un écart qui est à peu près de 2 décibels; le décibel étant vingt fois le logarithme du gain, cet écart correspond à peu près à une erreur de $20 \%$.

Au-dessous, nous avons la courbe de phase. Au lieu de $90^{\circ}$, nous trouvons ici que le vannage est légerement en avance sur sa valeur theorique, et cette avance du vamaze est variable avec la pulsation.

Le graphique $n^{\circ} 12$ est relatif aux essais a 
Fia. 14 Gain et déphasage du servo-moteur. Essai à $35 \mathrm{MW}$.

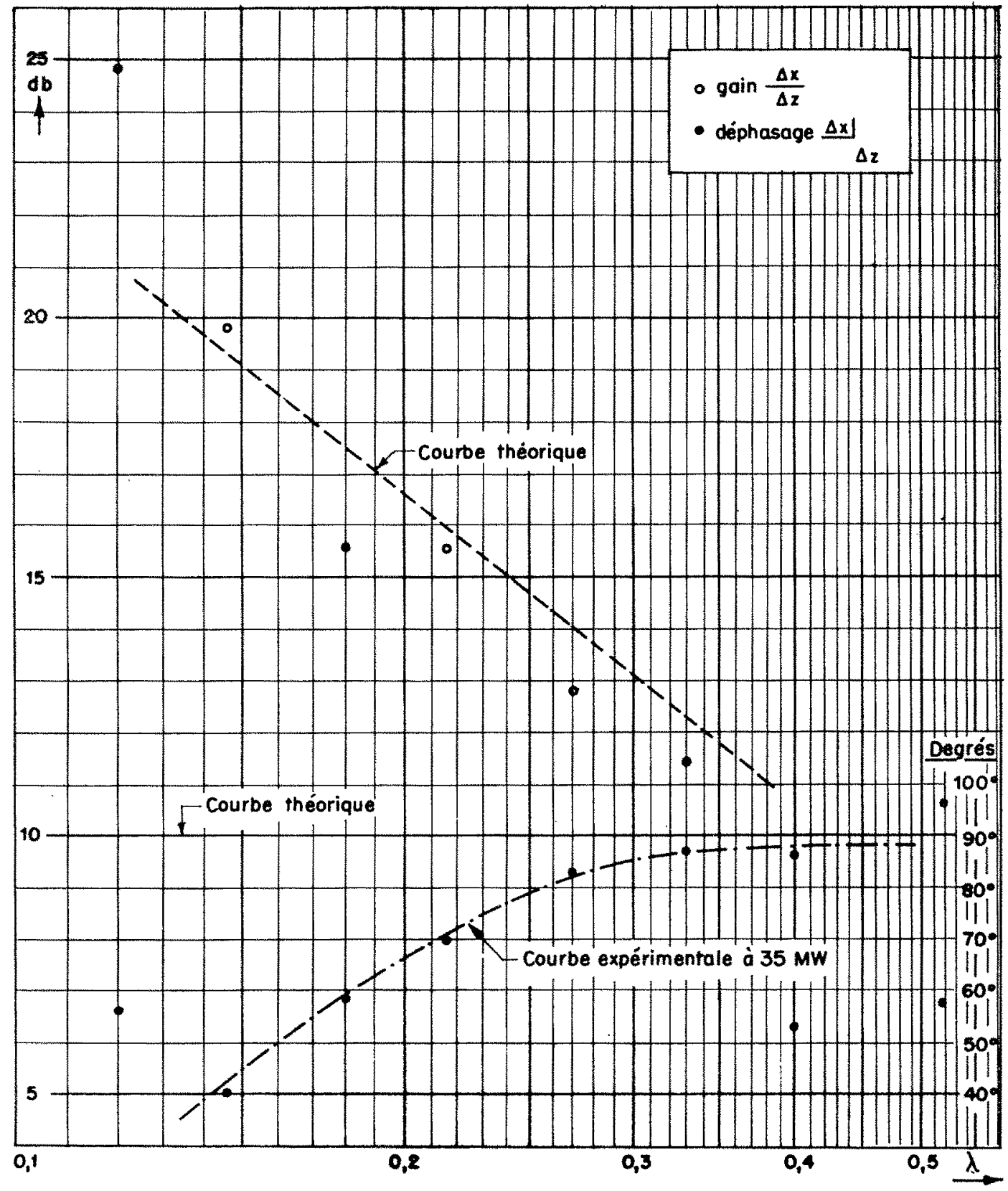

$15 \mathrm{MW}$. Ici, la courbe théorique correspond bien au relevé d'essais, puisque la dispersion de 1 décibel représente $10 \%$. De même la courbe de phase suit, aux dispersions près, c'est-à-dire à $10^{\circ}$, la courbe théorique; on trouve à peu près $90^{\circ}$ de déphasage.

Les courbes suivantes relatives aux essais à $25 \mathrm{MW}$ et a $35 \mathrm{MW}$ ont des caractéristiques voisines. La courbe théorique de gain coincide avec les résultats d'essais. Par contre, on observe entre les courbes de phase un écart qui croît à mesure que la pulsation diminue.

L'écart est assez important aux faibles pulsations.

Nous avons émis différentes hypothèses pour expliquer ce retard du vannage, On peut par exemple envisager la poussée hydraulique sur les aubes du distributeur.

Considérons le diagramme qui représente le couple à fournir pour fermer le vannage en fonction de l'ouverture de celui-ci (fig. 15). Le constructeur réalise une légère sollicitation des aubes, à l'ouverture pour les faibles puissances, à la fermeture pour les fortes charges.

Si nous prenons, par exemple, une courbe de fonctionnement à faible charge, c'est-à-dire pour une faible ouverture du vannage, on voit que, plus on a tendance à ouvrir le vannage, plus le couple négatif descend; par conséquent, il faut, 


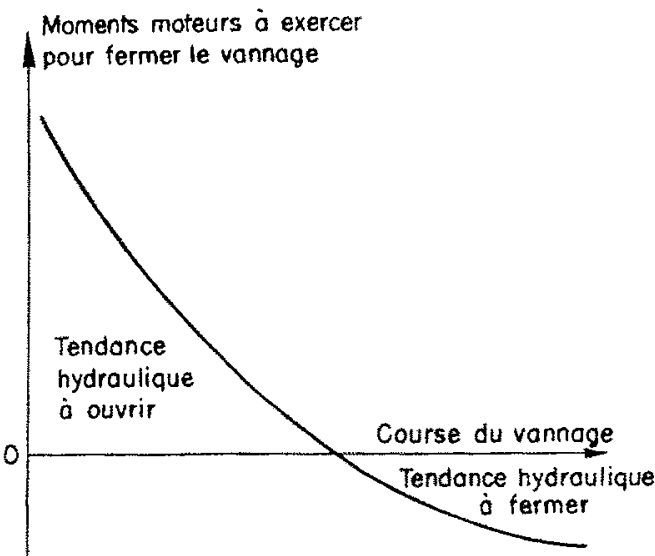

Fra. 15. - Influence de la poussée hydraulique sur Ies efforts a fournir pour fermer le vannage. en valeur absolue, fournir un couple plus grand pour l'ouvrir.

Ceci a donc un effet analogue à celui d'un stalisme positif qui pourrait, à la rigueur, expliquer l'avance qu'on a trouvée à vide pour les cearts. Mais il faudrait, pour expliquer le retard à 25 et $35 \mathrm{MW}$, admettre aux fortes charges une remontée de la courbe de couple, ce qui n'est pas réalisé en général.

On a essayé de l'expliquer aussi par des influences de coups de bélier, d'huile dans le servomoteur. Ces hypothèses ne nous paraissent pas pouvoir expliquer la forme des courbes de déphasage obtenues.

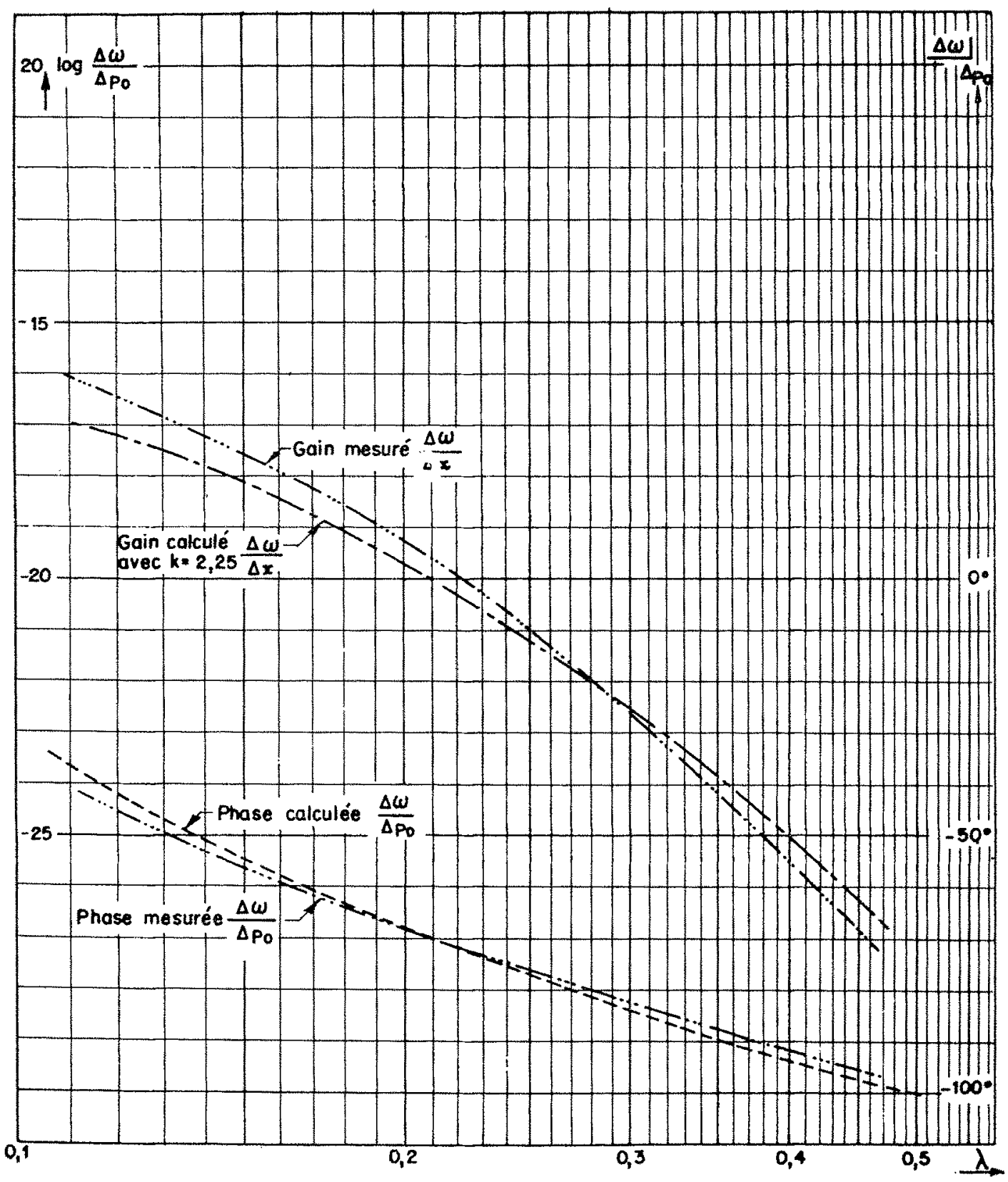

Fik: 16

Gain et dephasage de la

seconde partic

de Ja chaîne.

Essai à 15 MW. 
Fig. 17

Gain et déphasage de la seconde partie de la chaîne. Essai à 25 MW.

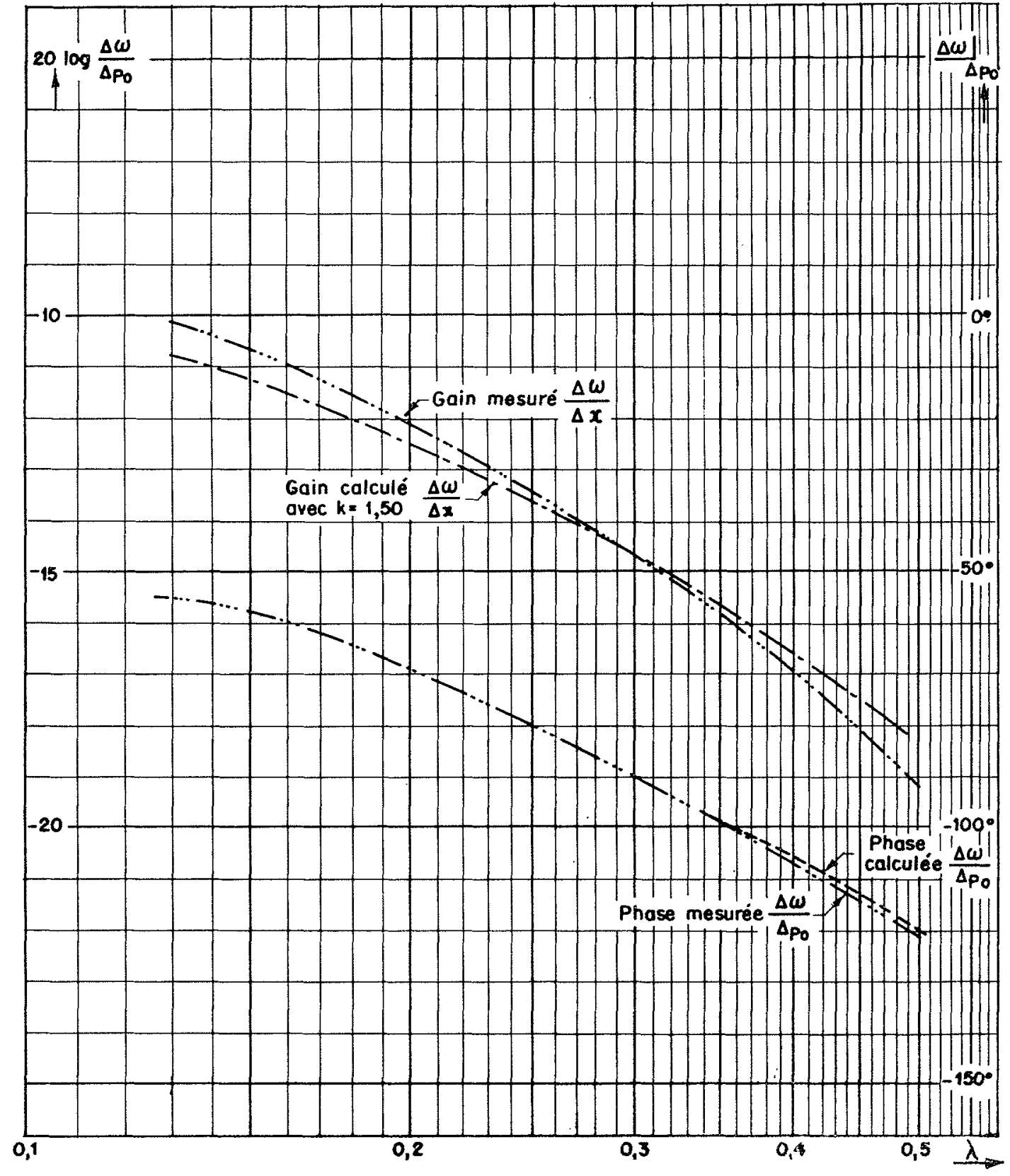

\section{B) Etude de la seconde partie de la chaîne :}

Cette seconde partie est caractérisée par les rapports $\Delta p / \Delta p_{0}$ (influence du coup de bélier) et $\Delta \omega / \Delta p$ (réaction des masses tournantes). L'écart n'a pu être relevé puisqu'il s'agit d'un ćcart de puissance théorique à pression constante. On peut cependant l'obtenir à partir de l'enregistrement du vamnage puisque :

$$
\Delta p_{0}=\frac{\Delta x}{K}
$$

K étant l'inverse de la pente de la courbe puissance-ouverture. 


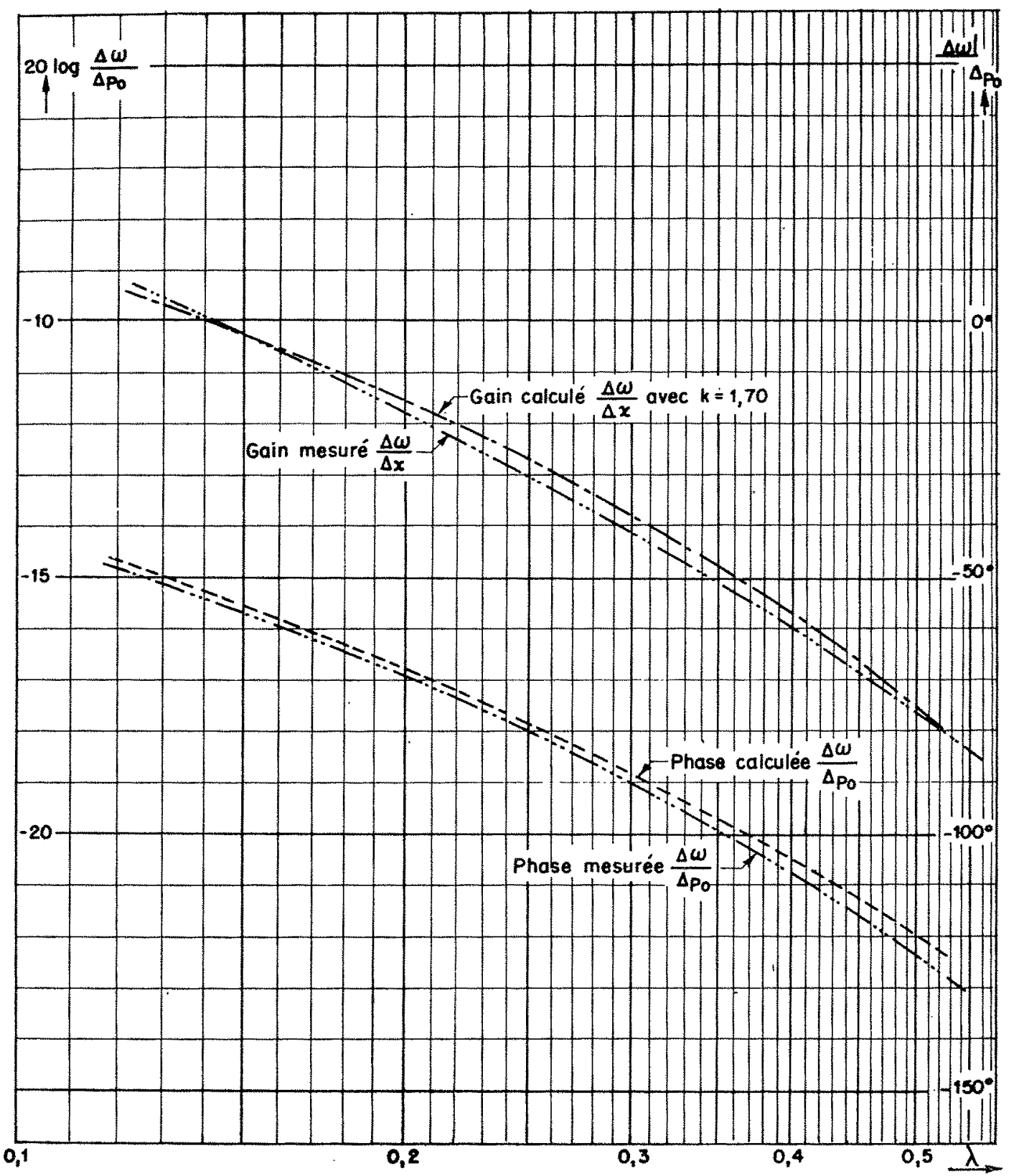

Fic. 18

Gain et déphasage de la seconde partic de la chaîne. Essai à 35 MW.

L'écart $\Delta p$ n'ayant pu être enregistré, nous comparerons les courbes théoriques et expérimentales représentant les effets globaux des deux éléments (coup de bélier et masses tournantes).

Les figures 16-17-18 montrent les résultats oblenus à 15,25 et $35 \mathrm{MW}$. On voit que les courbes théoriques et expérimentales sont très voisines.
Par conséquent, en conclusion de ces essais, si le fonctionnement du régleur paraît très correct, le servo-moteur, au contraire, introduit aux fortes charges un déphasage, dont nous n'avons pu déceler l'origine.

Les équations admises pour le coup de bélier et les masses tournantes semblent bien vérifiées. 


\section{IV. - INFLUENCE SUR LA STABILITÉ DES RETARDS OBSERVÉS}

Afin de montrer d'une façon concrète l'influence des retards constatés, nous avons, bien que ce ne soit pas le but de l'étude, étudié la stabilité globale du groupe.

Dans ce but, on relève le gain $\Delta \omega / \Delta \omega_{0}$ de la chaine fermée. On peut ensuite opérer de deux manières différentes :

$1^{\circ}$ Soit calculer les caractéristiques de la chaîne ouverte à l'aide d'abaques comme le diagramme de Black. On obtient alors un critère de stabilité défini par le complément à $180^{\circ} \mathrm{du}$ déphasage de la chaine ouverte pour la pulsation qui donne un gain égal à l'unité (marge de phase).

Le tableau de la figure 19 représente la marge de phase trouvée à la suite des essais et à la suite du calcul. A $15 \mathrm{MW}$, on voit que la marge de phase expérimentale et la marge de phase calculée sont identiques. Par conséquent, comme nous l'avons déjà fait remarquer, le servomoteur suit la loi théorique et il est normal de retrouver la même stabilité.

Par contre, pour les essais à forte puissance : 25 et $35 \mathrm{MW}$, la marge de phase est beaucoup plus faible que celle qui était prévue. A $25 \mathrm{MW}$, on perd de 10 à $12^{\circ}$; à $35 \mathrm{MW}$, on perd $18^{\circ}$.

Par conséquent, ceci montre l'influence néfaste d'un retard du vannage sur la stabilité.

$2^{\circ}$ Pour préciser l'influence de cette marge de phase, nous allons étudier l'amortissement des oscillations, que l'on peut obtenir à partir de la courbe de gain en chaîne fermée, par des méthodes graphiques approchées.
Pour cela, on suppose que l'équation des oscillations de vilesse est toujours de la forme:

$$
\frac{d^{2} \Delta \omega}{d t^{2}}+\mathrm{P} \frac{d}{d t} \Delta \omega+\mathrm{Q} \Delta \omega=0
$$

et on détermine, à partir du gain de la chaîne fermée, les coefficients $P$ et $Q$.

Le coefficient $P$ mesure l'amortissement. Si l'on donne un à-coup de vitesse, par exemple au moyen du variateur, la fréquence va suivre une courbe semblable à celle qu'indique la figure 20 ; l'enveloppe de cette courbe est une exponentielle de forme $\mathrm{A} e^{-p / 2} \mathrm{q}^{\prime \prime}$, où $\mathrm{T}^{\prime \prime}$ est la pseudo-période.

Pour éliminer les oscillations parasites, on avait rendu le groupe très stable en augmentant l'autoréglage. Il a donc paru plus précis de déterminer $\mathrm{P}$ à partir des relations approchées de la figure 20.

D'autre part, le coefficient $Q$ mesure la période d'oscillations naturelles.

L'équation précédente est relative à un groupe seul, mais si plusieurs groupes fonctionnent en parallèle, on peut, en première approximation, assimiler l'ensemble à une seule machine obéissant à une équation de la même forme. Il est possible de calculer les coefficients $P$ et $Q$ relatifs à l'ensemble à partir des coefficients de chacun des groupes d'une façon très simple.

Ces grandeurs peuvent done être considérées comme des paramètres qui, associés à un coefficient de pondération, définissent une machine au point de vue de la stabilité.

\begin{tabular}{|c|c|c|c|c|c|c|c|c|}
\hline \multirow{2}{*}{$\begin{array}{l}\text { Puissance } \\
\text { du } \\
\text { groupe MW }\end{array}$} & \multirow{2}{*}{$\begin{array}{c}\text { Asservissement } \\
\text { Tension } \\
\text { fréquence }\end{array}$} & \multirow{2}{*}{$\alpha$} & \multicolumn{2}{|c|}{$\begin{array}{l}\text { Temps caractéristique } \\
\text { de la promptitude }\end{array}$} & \multirow{2}{*}{$m$} & \multirow{2}{*}{$\Theta$} & \multirow{2}{*}{$j$} & \multirow{2}{*}{$\mathrm{T}$} \\
\hline & & & $\tau_{y}^{\prime}$ & $\tau^{\prime}=\tau_{z}^{\prime} \cdot \mathrm{K}$ & & & & \\
\hline \multirow{2}{*}{15} & manuel & 2,4 & 0,40 & 0,90 & 2,70 & 0,69 & 1,06 & 18,5 \\
\hline & +1 & 1,69 & 0,40 & 0,90 & 2,70 & 0,69 & 1,06 & 18,5 \\
\hline \multirow{2}{*}{25} & manuel & 1,97 & 0,60 & 0,90 & 2,70 & 1,18 & 1 & 11,4 \\
\hline & +1 & 1,50 & 0,60 & 0,90 & 2,70 & 1,18 & 1 & 11,4 \\
\hline 35 & manuel & 1,62 & 0,73 & 1,25 & 2,70 & 1,60 & 1,4 & 8,25 \\
\hline
\end{tabular}

FIG. 19 


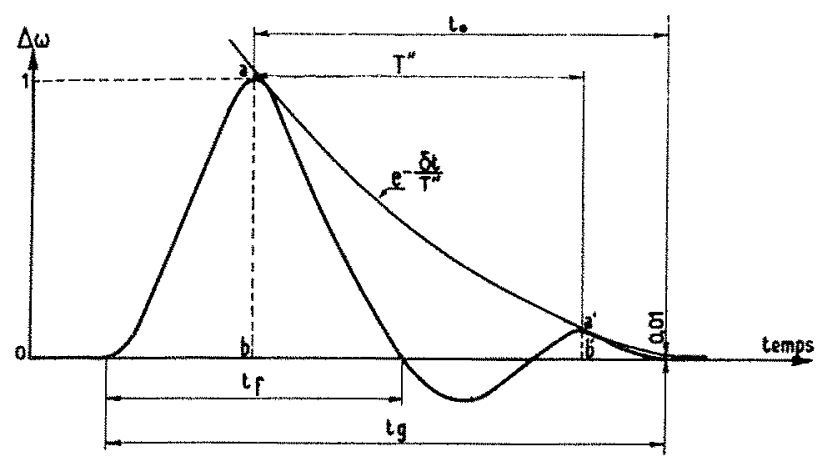

a) Oscillation à la suite d'un à coup.

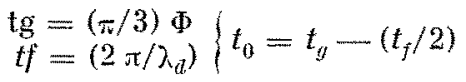

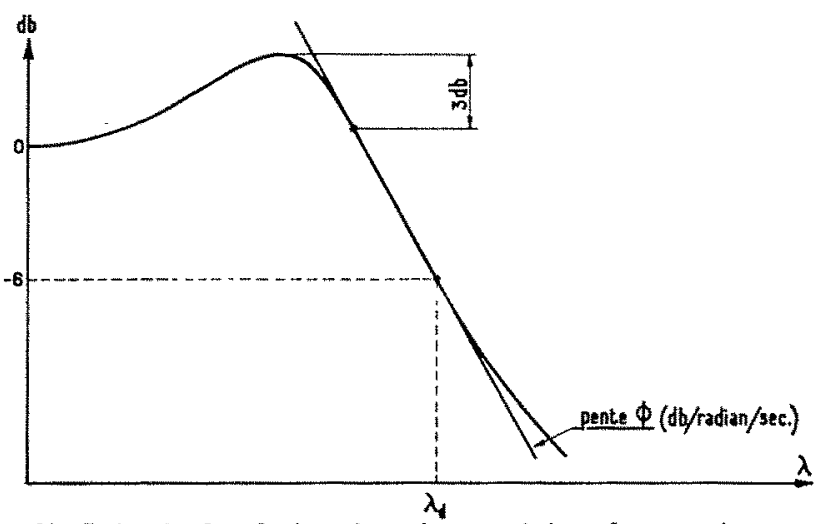

b) Gain de la chaîne fermée en régime harmonique.

$\mathrm{P}=\left(2 \delta / \mathrm{T}^{\prime \prime}\right)=2(\log 100) / t_{0}$

$\hat{o}=\log \left(a b / a^{\prime} b^{\prime}\right)$

Fic. 20 Relation entre les régimes transitoire et harmonique.

I] nous a donc paru intéressant de reporter les points figurant les groupes sur un diagramme en $(P, Q)$ en retenant que le coefficient $P$ peut être considéré comme mesurant la stabilité (fig. 21).

Les essais 13 et 14 sont relatifs à une puissance de $15 \mathrm{MW}$. On voit que la stabilite mesurée par l'abscisse suit à peu près les données théoriques.
Par contre, pour les essais 23 et 24 , à $25 \mathrm{MW}$, et pour l'essai 31 , à $35 \mathrm{MW}$, c'est-à-dire pour les essais à forte charge, les points d'essai sont décalés vers la gauche par rapport aux points correspondant au fonctionnement théorique du régulateur.

La réduction de stabilité entrainée par le retard du vannage peut donc être estimée avec ce mode de représentation.

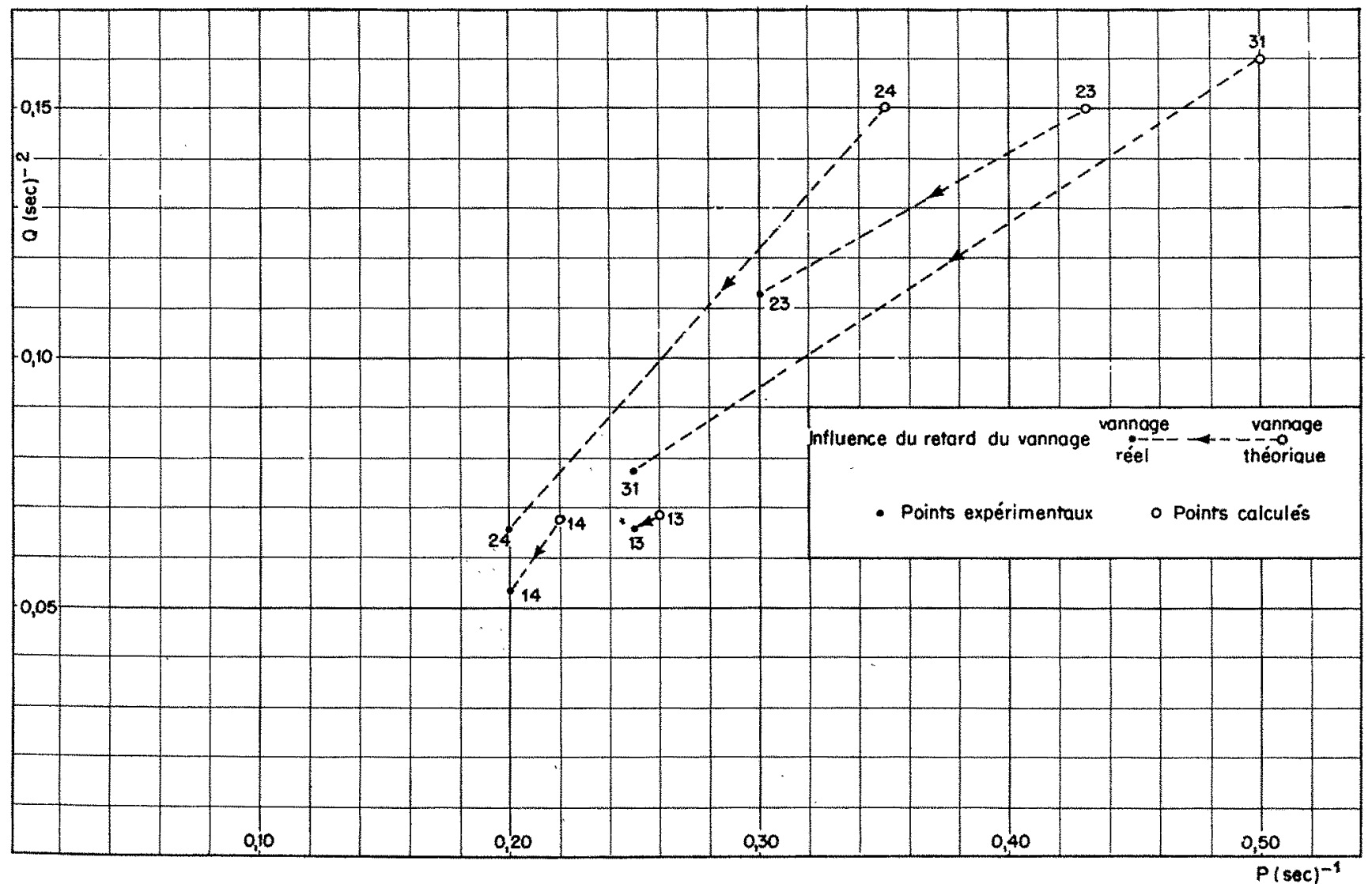

Fic. 21. - Diagramme (PQ) indiquant l'infuence sur la stabilite du retard du vannage. 
Par exemple, pour l'essai 24, le rapport théoricue de deux amplitudes successives est égal à 36. On a donc une très bonne stabilité. Ce rapport est réduit à 8 pour le point expérimental. Par conséquent, on a une baisse très sensible de stabilité due uniquement au retard du vannage puisque pour la puissance de $15 \mathrm{MW}$ ce phénomène n’existe pas.
Le diagramme met aussi en relief l'influence de l'autoréglage sur la stabilité. C'est ainsi que les essais 13 et 23 à régulateur de tension bloqué indiquent une plus grande stabilité que les essais 14 et 24 eflectués aux mêmes puissances mais avec un régulateur de tension en service (asservissement maximum).

\section{CONCLUSION}

Les essais ont montré que, d'une façon générale, les équations de la régulation sont vérifiées, sauf en ce qui concerne le servo-moteur, et les essais ultérieurs auront donc à porter spécialement sur cet élément.

On peut remarquer d'ailleurs que, pour étudier ce point particulier, il ne sera pas nécessaire de fonctionner sur résistance liquide.

Le groupe étant couplé sur le réseau, on peut toujours, au moyen du changement de vitesse, agir sur le vannage de facon sinusoïdale.

Les essais semblent d'autant plus faciles à ef- fectuer sur résean que l'on ne sera pas gêné ni par les perturbations de la résistance liquide, qui nous ont limité aux grandes périodes, ni par l'inertie des masses tournantes qui atténue le gain aux faibles périodes.

Par conséquent, on peut espérer explorer un domaine de pulsations plus grand.

Il y aurait lieu en outre de prévoir une deuxième série d'essais au cours de laquelle pourrait être étudié le fonctionnement de plusieurs groupes en parallèle, ce qui n'a pas été possible par suite des exigences d'exploitation à Ottmarsheim.

\section{DISCUSSION}

Irésident : M. BanRuLION

M. le Président remercie et félicite $M$. Launenr de sa communication, présentée d'une laçon tout à fait claire, et de son mémoire très bien rédigé.

Parmi les extensions que l'on fera incessamment, probablement, pour la mise en parallele, M. le Président pense qu'il serait bien intéressant de fiire un essai sur une station ayant une hauteur de chute plus grande.

M. Bonnis voudrait mettre l'accent sur la linéarité de la correspondance entre deux gronpes fonctionnant en parallèle, linéarité qui permet - c'est l'idée originale de cette étude - de chiffrer une réserve de stabilité composée de l'ensemble.

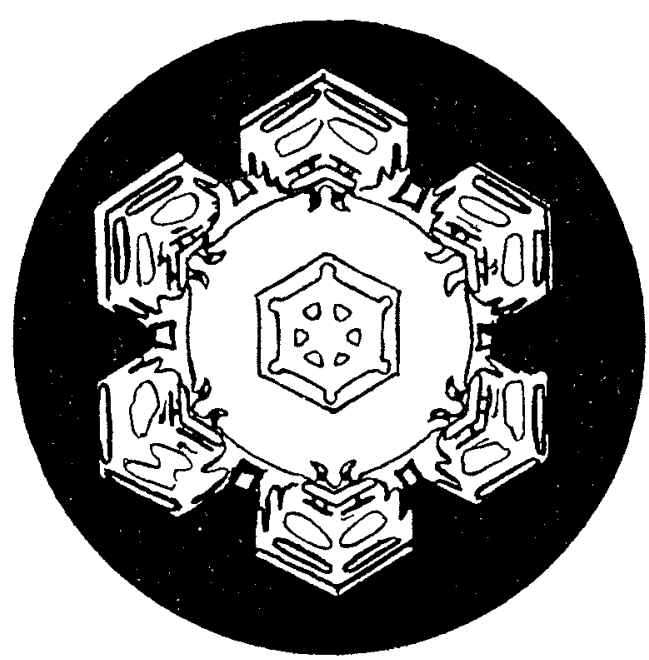

